\title{
In situ Formed Catalytic Interface for Boosting Chemiluminescence
}

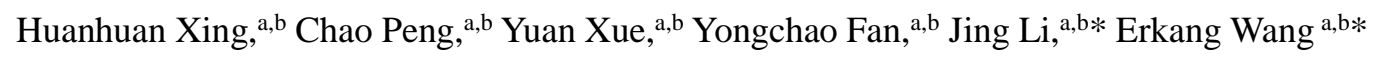

a. State Key Laboratory of Electroanalytical Chemistry, Changchun Institute of Applied Chemistry, Chinese Academy of Sciences, Changchun, Jilin, 130022, China.

b. University of Science and Technology of China, Hefei, Anhui, 230026, P. R. China.

Corresponding author: Tel:+86-431-85262003, Fax: +86-431-85689711, E-mail:

lijingce@ciac.ac.cn; ekwang@ciac.ac.cn

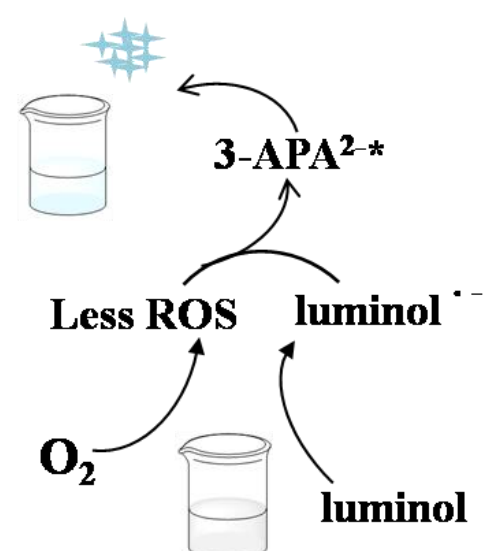

BSA or $\mathrm{Co}^{2+}$

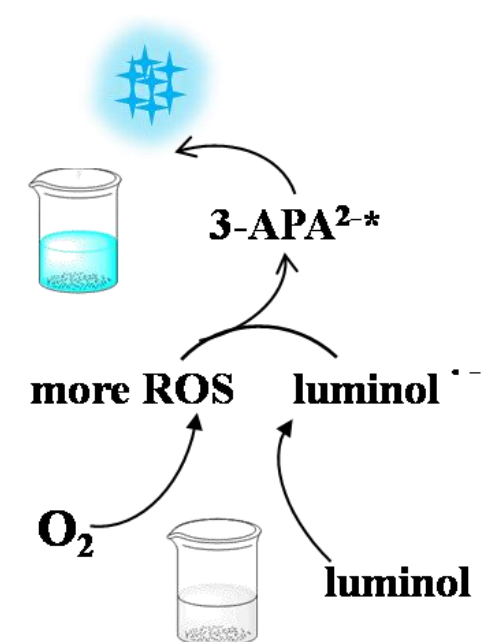

BSA and $\mathrm{Co}^{2+}$

Scheme S1: The experimental schematic diagram of the enhanced CL of luminol mediated by $\mathrm{BSA}$ and $\mathrm{Co}^{2+}$ in alkaline condition. 


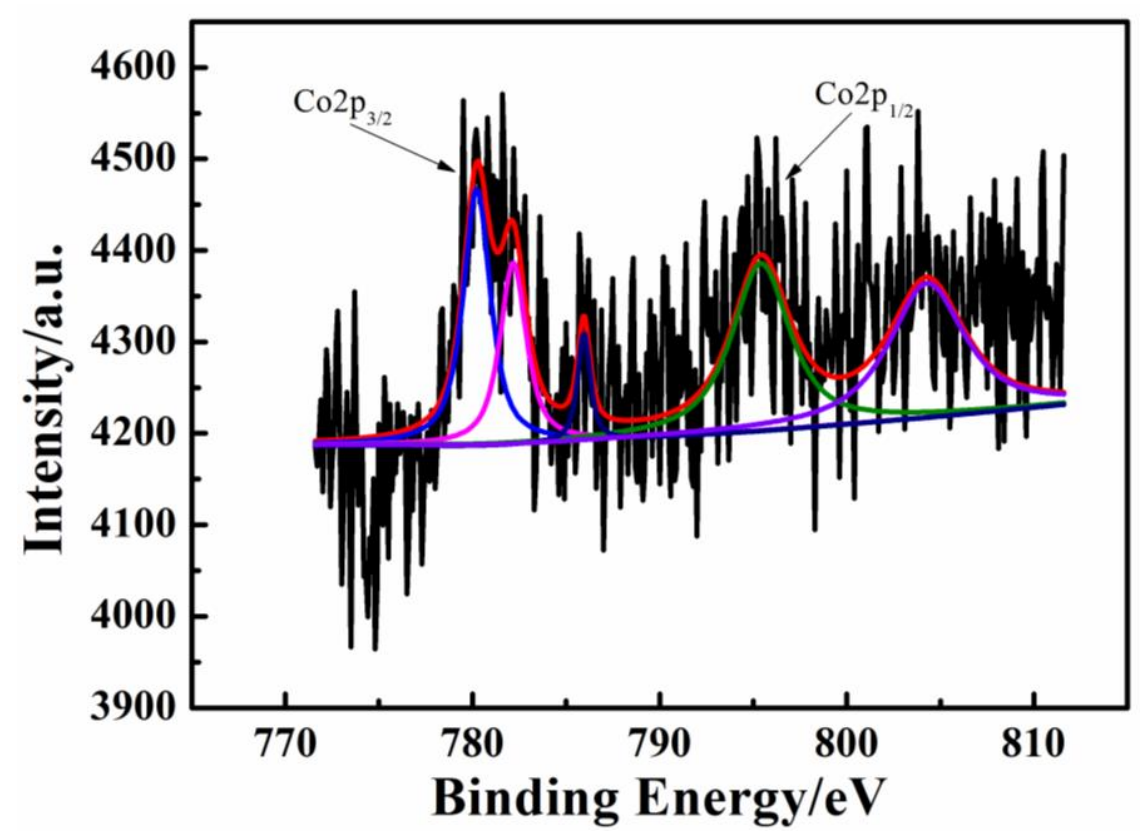

Figure S1: The XPS of Co element of the in situ generated $\mathrm{f}-\mathrm{Co}(\mathrm{OH})_{2}$.
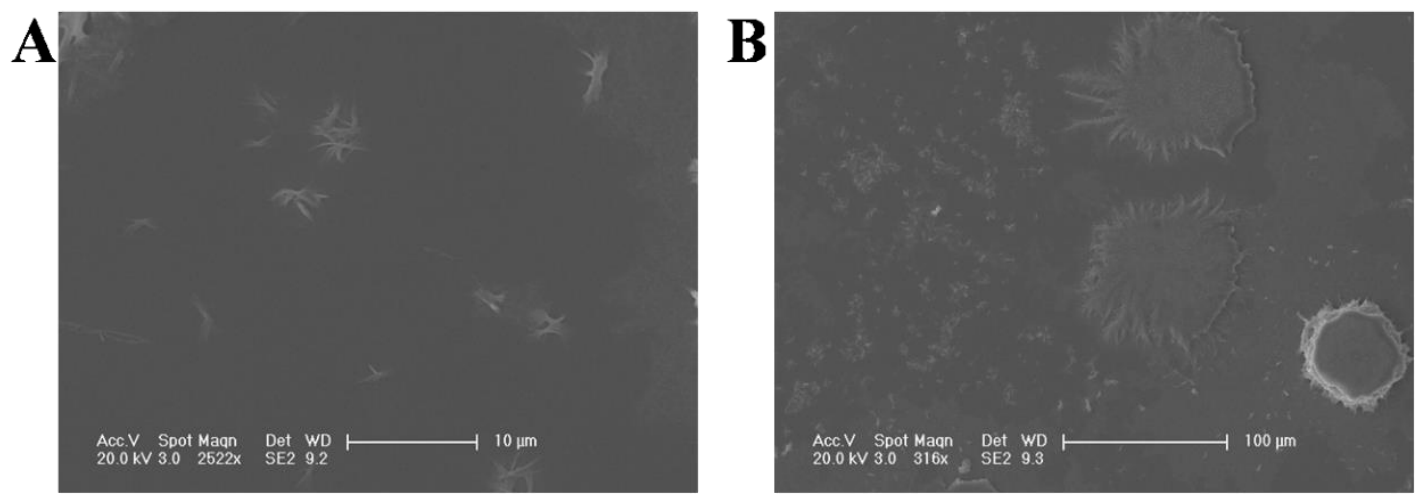

Figure S2: The SEM image of b-Co(OH $)_{2}$ in alkaline solution in the different magnification.

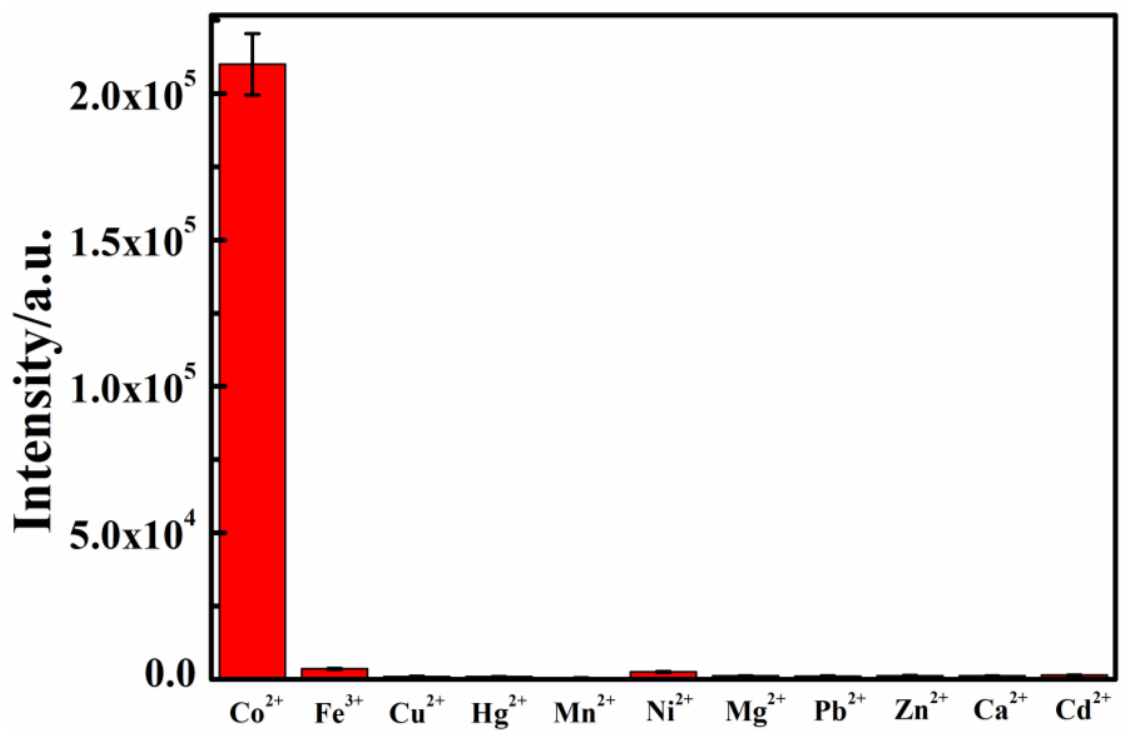

Figure S3: The CL response of luminol-BSA system using different metal ions with the same concentration. 


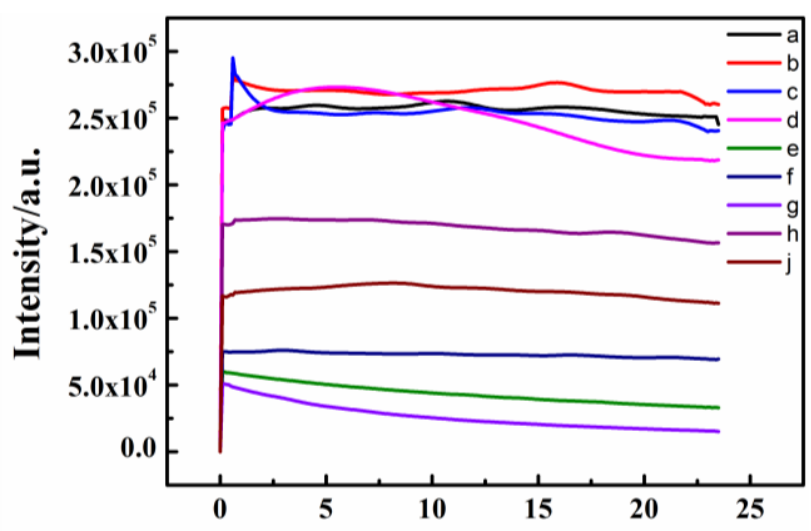

Time/s

Figure S4: The CL kinetic curves induced by different proteins $(0.2 \mathrm{mg} / \mathrm{mL})$ in $100 \mu \mathrm{M} \mathrm{Co}^{2+}$ and 3 mM luminol at pH 11.5 PBS. a (BSA), b (lysozyme), c (ConA), d (papain), e (thrombin), f (bovine hemoglobin), g (glucose oxidase), h (lactoglobulin), j (protamine).
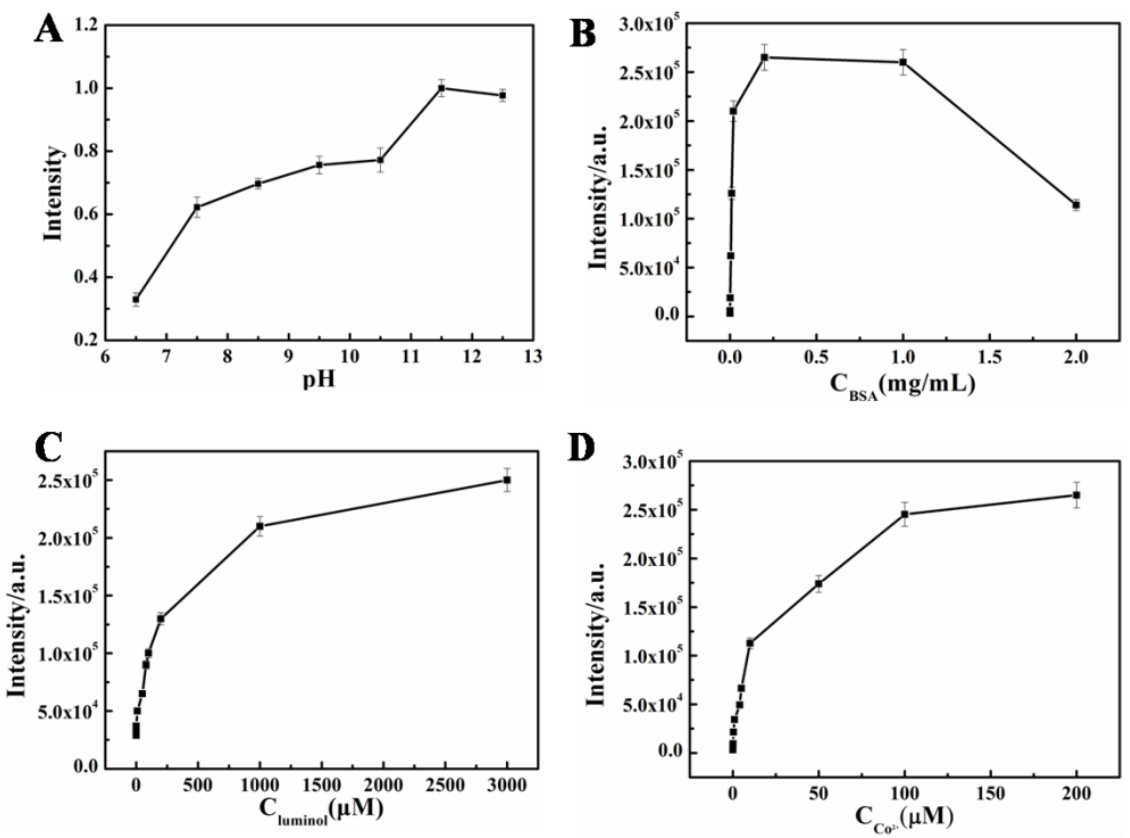

Figure S5: The optimization of $\mathrm{pH}(\mathrm{A})$, the concentration of BSA (B), luminol (C) and $\mathrm{Co}^{2+}$ (D) based on the proposed CL system.

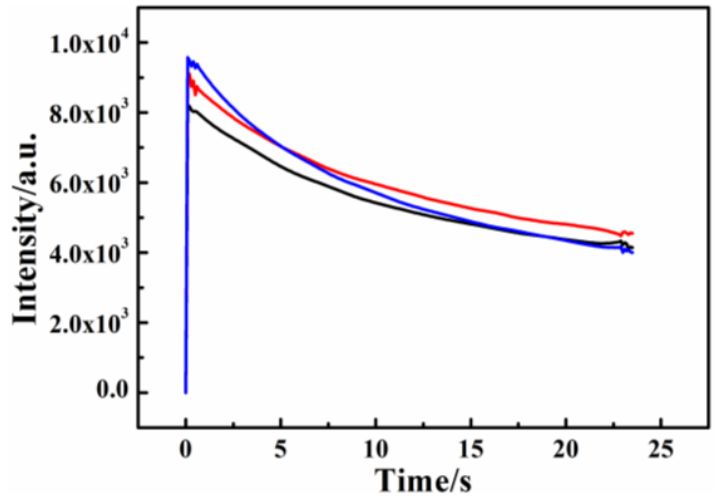

Figure S6: The reproducibility experiment of the proposed CL sensor for the detection of $25 \mu \mathrm{M}$ AA. 


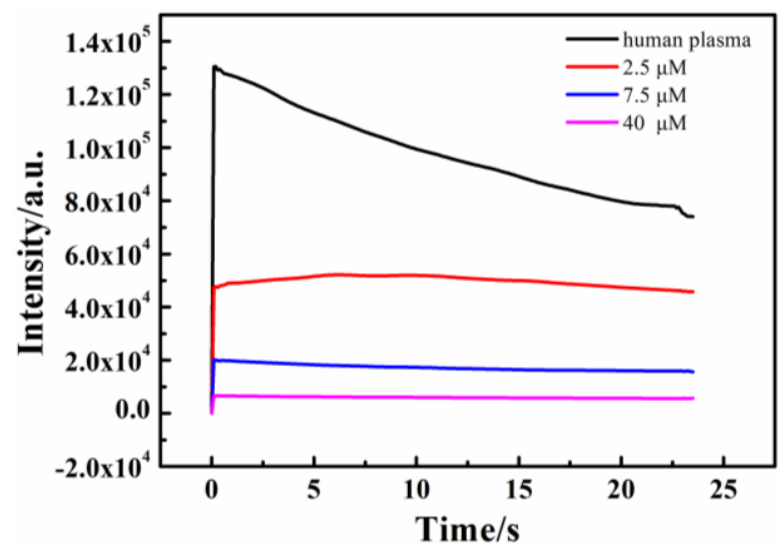

Figure S7: The CL kinetic curves based on the proposed sensor with the additional different concentration of AA in human serum.

Table S1: The recovery for the detection of AA in human serum

\begin{tabular}{cccc}
\hline sample & AA added & AA detected & recovery \\
\hline $\mathbf{1}$ & $2.5 \mu \mathrm{M}$ & $2.95 \mu \mathrm{M}$ & $95.1 \%$ \\
$\mathbf{2}$ & $7.5 \mu \mathrm{M}$ & $8.12 \mu \mathrm{M}$ & $100.2 \%$ \\
$\mathbf{3}$ & $40 \mu \mathrm{M}$ & $42.6 \mu \mathrm{M}$ & $92.6 \%$ \\
\hline
\end{tabular}

